CUBO A Mathematical Journal

Vol.14, No 01, (29-47). March 2012

\title{
Spectral shift function for slowly varying perturbation of periodic Schrödinger operators.
}

\author{
Mouez Dimassi \\ Univ. Paris 13, \\ LAGA, (UMR CNRS 7539), \\ F-93430 Villetaneuse, France, \\ email: dimassi@math.univ-paris13.fr \\ and \\ MAHER ZERZERI \\ Univ. Paris 13, \\ LAGA, (UMR CNRS 7539), \\ F-93430 Villetaneuse, France, \\ email: zerzeri@math.univ-paris13.fr
}

\begin{abstract}
In this paper we study the asymptotic expansion of the spectral shift function for the slowly varying perturbations of periodic Schrödinger operators. We give a weak and pointwise asymptotic expansions in powers of $h$ of the derivative of the spectral shift function corresponding to the pair $\left(\mathrm{P}(\mathrm{h})=\mathrm{P}_{0}+\varphi(h x), \mathrm{P}_{0}=-\Delta+\mathrm{V}(\mathrm{x})\right)$, where $\varphi(x) \in \mathcal{C}^{\infty}\left(\mathbb{R}^{n}, \mathbb{R}\right)$ is a decreasing function, $\mathcal{O}\left(|x|^{-\delta}\right)$ for some $\delta>n$ and $h$ is a small positive parameter. Here the potential $\mathrm{V}$ is real, smooth and periodic with respect to a lattice $\Gamma$ in $\mathbb{R}^{n}$. To prove the pointwise asymptotic expansion of the spectral shift function, we establish a limiting absorption Theorem for $\mathrm{P}(\mathrm{h})$.
\end{abstract}




\section{RESUMEN}

En este artículo estudiamos la expansión asintótica de la función shift espectral para perturbaciones de variación lenta de operadores periódicos de Schrödinger. Proporcionamos una expansión débil y puntual en potencias de $h$ de la derivada de la función shift espectral que corresponde al $\operatorname{par}\left(\mathrm{P}(\mathrm{h})=\mathrm{P}_{0}+\varphi(\mathrm{h} x), \mathrm{P}_{0}=-\Delta+\mathrm{V}(\mathrm{x})\right)$, donde $\varphi(x) \in \mathcal{C}^{\infty}\left(\mathbb{R}^{n}, \mathbb{R}\right)$ es una función decreciente, $\mathcal{O}\left(|x|^{-\delta}\right)$ para algún $\delta>n \mathrm{y} h$ un parámetro positivo pequeño. Aquí el potencial $\mathrm{V}$ es real, suave y periódico con respecto a un retículo $\Gamma$ en $\mathbb{R}^{n}$. Para demostrar la expansión asintótica puntual de la función shift espectral establecemos un teorema de absorción límite para $\mathrm{P}(\mathrm{h})$.

Keywords and Phrases: Periodic Schrödinger operator, spectral shift function, asymptotic expansions, limiting absorption theorem.

2010 AMS Mathematics Subject Classification: 81Q10 (35P20 47A55 47N50 81Q15)

\section{Introduction}

The aim of this paper is to give an asymptotic expansion of the spectral shift function for the slowly varying perturbations of periodic Schrödinger operator:

$$
\begin{gathered}
P(h)=P_{0}+\varphi(h x), \quad h>0, \\
P_{0}=-\Delta_{x}+V(x),
\end{gathered}
$$

Here $\mathrm{V}$ is a real-valued, $\mathcal{C}^{\infty}$ function and periodic with respect to a lattice $\Gamma$ of $\mathbb{R}^{n}$.

The Hamiltonian $\mathrm{P}(\mathrm{h})$ describes the quantum motion of an electron in a crystal placed in an external field. There are many works devoted to the spectral properties of this model, see $[1,3,5,8,9,10,12,13,15,16,18,21,36]$.

We assume that $\varphi \in \mathcal{C}^{\infty}\left(\mathbb{R}^{n} ; \mathbb{R}\right)$ and satisfies the following estimate: for all $\alpha \in \mathbb{N}^{n}$, there exists $C_{\alpha}>0$ such that

$$
\left|\partial_{x}^{\alpha} \varphi(x)\right| \leq C_{\alpha}(1+|x|)^{-\delta-|\alpha|}, \quad \forall x \in \mathbb{R}^{n}, \quad \text { with } \delta>n .
$$

The operators $P_{0}, P(h)$ are self-adjoint on $H^{2}\left(\mathbb{R}^{n}\right)$. Under the assumption (1.2) we show in Theorem 2.2 below that the operator $\left[f(P(h))-f\left(P_{0}\right)\right]$ belongs to the trace class for all $f \in \mathcal{C}_{0}^{\infty}(\mathbb{R})$. Following the general setup we define the spectral shift function, SSF, $\xi_{h}(\mu):=\xi\left(\mu ; P(h), P_{0}\right)$ related to the pair $\left(\mathrm{P}(\mathrm{h}), \mathrm{P}_{0}\right)$ by

$$
\operatorname{tr}\left[\mathrm{f}(\mathrm{P}(\mathrm{h}))-\mathrm{f}\left(\mathrm{P}_{0}\right)\right]=-\left\langle\xi_{h}^{\prime}(\cdot), f(\cdot)\right\rangle=\int_{\mathbb{R}} \xi_{h}(\mu) f^{\prime}(\mu) \mathrm{d} \mu, \quad \forall f \in \mathcal{C}_{0}^{\infty}(\mathbb{R}) .
$$

By this formula $\xi_{h}$ is defined modulo a constant but for the analysis of the derivative $\xi_{h}^{\prime}(\mu)$ this is not important. See [22] and [2]. 
The SSF may be considered as a generalization of the eigenvalues counting function. The notion of SSF was first singled out by the outstanding theoretical physicist I-M.Lifshits in his investigations in the solid state theory, in 1952, see [23]. It was brought into mathematical use in M-G. Kreln's famous paper [22], where the precise statement of the problem was given and explicit representation of the SSF in term of the perturbation determinant was obtained. The work of M-G. Kreĭn's on the SSF has been described in detail in [2]. For more details about the interpretation of SSF we refer to the survey by D. Robert [32] and to the monograph by D-R. Yafaev [42, Chapter $8]$.

In the case where $\mathrm{V}=0$, the asymptotic behavior of the SSF of the Schrödinger operator has been intensively studied in different aspects (see [6, 17, 24, 25, 30, 31, 34] and the references given there). In the semi-classical regime (i.e. $H(h)=-h^{2} \Delta_{x}+\varphi(x),(h \searrow 0)$ ) the Weyl type asymptotics of $\xi_{h}(\cdot)$ with sharp remainder estimate has been obtained (see [30,31,34,35]). On the other hand, if an energy $\mu>0$ is non-trapping for the classical hamiltonian $p(x, \zeta)=|\zeta|^{2}+\varphi(x)$ (i.e. for all $(x, \zeta) \in p^{-1}\{\mu\}$, $\left|\operatorname{expt}_{\mathrm{p}}(x, \zeta)\right| \rightarrow \infty$ when $\left.t \rightarrow \infty\right)$ a complete asymptotic expansion in powers of $h$ of $\xi_{h}^{\prime}(\mu)$ has been obtained (see [30,31,34,35]). Similar results are well-known for the SSF at high energy (see $[4,6,25,26,29]$ ).

There are only few works treating the SSF in perturbed periodic Schrödinger operator. See [3], [10] and also [16]. In [10] the connection between the resonances of $\mathrm{P}(\mathrm{h})$ and the SSF associated to the pair $\left(\mathrm{P}(\mathrm{h}), \mathrm{P}_{0}\right)$ were studied. Under the assumption that $\varphi$ is analytic in some conic complex neighborhood of the real axis and that $\mathrm{P}(\mathrm{h})$ has no resonances in a small complex neighborhood of some interval I the first author obtained a full asymptotic expansion in powers of $h$ of the derivative of SSF:

$$
\xi^{\prime}(\mu ; h) \sim \sum_{j=0}^{\infty} b_{j}(\mu) h^{j-n}, \quad h \searrow 0,
$$

uniformly with respect to $\mu \in \mathrm{I}$.

Nevertheless, there is a lot of examples of perturbed periodic Schrödinger operator that the perturbation $\varphi$ does not satisfies the analyticity assumption.

In this paper, we improve the result of [10] concerning the behavior of the derivative of SSF by removing the analyticity assumption on the potential $\varphi$. Our proof is based on a limiting absorption principle and some arguments due to D. Robert and H. Tamura, see [33] and [35]. For $\mathrm{V} \neq 0$, the limiting absorption theorem is new (see Theorem 3.11). They are in harmony with the physical intuition which argues that, when $h$ sufficiently small, the main effect of the periodic potential $\mathrm{V}$ consists in changing the dispersion relation from the free kinetic $|\mathrm{k}|^{2}$ to the modified kinetic energy $\lambda_{p}(k)$ given by the pth band.

By the method of effective hamiltonian spectral problems of $\mathrm{P}(\mathrm{h})$ can be reduced to similar problem of system of $h$-pseudodifferential operators (See [9] and also [12]). Using a well-known results on $h$-pseudodifferential calculus we get the asymptotic (1.4) in the sense of distributions. If the values of the principal term of the effective hamiltonian are contained in non-trapping energy region we prove a limiting absorption principle for $\mathrm{P}(\mathrm{h})$ (Theorem 3.11) and we get a pointwise 
asymptotic expansion for the derivative of the spectral shift function.

The paper is organized as follows: In the next section, we recall some well-known results concerning the spectra of a periodic Schrödinger operator (subsection 2.1) and we state the assumptions and the results precisely (subsection 2.2). We give an outline of the proofs in subsection 2.3. Section 3 is devoted to the proofs. Roughly, we introduce a class of symbols and the corresponding $\mathrm{h}$-Weyl operators (subsection 3.1). In the subsection 3.2 we recall the effective Hamiltonian method and we give a representation of the derivative of the spectral shift function, denoted by $\zeta_{h}^{\prime}(\cdot)$. The proof of the weak asymptotic expansion of $\xi_{h}^{\prime}$ is given in subsection 3.3. We establish a limiting absorption principle for $\mathrm{P}(\mathrm{h})$ in the subsection 3.4 At last, the pointwise asymptotic expansion of $\xi_{h}^{\prime}$ is proved in subsection 3.5.

\section{Statements}

\subsection{Preliminaries}

Let $\Gamma=\bigoplus_{i=1}^{n} \mathbb{Z} e_{i}$ be a lattice generated by some basis $\left(e_{1}, e_{2}, \cdots, e_{n}\right)$ of $\mathbb{R}^{n}$. The dual lattice $\Gamma^{*}$ is given by $\Gamma^{*}:=\left\{\gamma^{*} \in \mathbb{R}^{n} ;\left\langle\gamma \mid \gamma^{*}\right\rangle \in 2 \pi \mathbb{Z}, \forall \gamma \in \Gamma\right\}$. A fundamental domain of $\Gamma$ (resp. $\Gamma^{*}$ ) is denoted by $E$ (resp. $E^{*}$ ). If we identify opposite edges of $E$ (resp. $E^{*}$ ) then it becomes a flat torus denoted by $\mathbb{T}=\mathbb{R}^{n} / \Gamma$ (resp. $\left.\mathbb{T}^{*}=\mathbb{R}^{n} / \Gamma^{*}\right)$.

Let $\mathrm{V}$ be a real-valued potential, $\mathcal{C}^{\infty}$ and $\Gamma$-periodic. For $k \in \mathbb{R}^{n}$, we define the operator $P(k)$ on $L^{2}(\mathbb{T})$ by $P(k):=\left(D_{y}+k\right)^{2}+V(y)$. The operator $P(k)$ is a semi-bounded self-adjoint with $k$-independent domain $H^{2}(\mathbb{T})$. Since the resolvent of $P(k)$ is compact, $P(k)$ has a complete set of (normalized) eigenfunctions $\Phi_{n}(\cdot, k) \in H^{2}(\mathbb{T}), n \in \mathbb{N}$, called Bloch functions. The corresponding eigenvalues accumulate at infinity and we enumerate them according to their multiplicities, $\lambda_{1}(k) \leq$ $\lambda_{2}(\mathrm{k}) \leq \cdots$. The operator $\mathrm{P}(\mathrm{k})$ satisfies the identity $e^{-i y \cdot \gamma^{*}} \mathrm{P}(\mathrm{k}) \mathrm{e}^{i \mathrm{y} \cdot \gamma^{*}}=\mathrm{P}\left(\mathrm{k}+\gamma^{*}\right), \forall \gamma^{*} \in \Gamma^{*}$, then for every $p \geq 1$, the function $k \mapsto \lambda_{p}(k)$ is $\Gamma^{*}$-periodic.

Ordinary perturbation theory shows that $\lambda_{p}(k)$ are continuous functions of $k$ for any fixed $p$, and $\lambda_{p}(k)$ is even an analytic function of $k$ near any point $k_{0} \in \mathbb{T}^{*}$ where $\lambda_{p}\left(k_{0}\right)$ is a simple eigenvalue of $P\left(k_{0}\right)$. The function $\lambda_{p}(k)$ is called the band function and the closed intervals $\Lambda_{p}:=$ $\lambda_{\mathrm{p}}\left(\mathbb{T}^{*}\right)$ are called bands. See [27], [39] and also [37, 38].

Consider the self-adjoint operator on $\mathrm{L}^{2}\left(\mathbb{R}^{n}\right)$ with domain $\mathrm{H}^{2}\left(\mathbb{R}^{n}\right)$ :

$$
P_{0}=-\Delta_{x}+V(x), \quad \text { where } \Delta_{x}=\sum_{j=1}^{n} \frac{\partial^{2}}{\partial x_{j}^{2}}
$$

The spectrum of $P_{0}$ is absolutely continuous (see [41]) and consists of the bands $\Lambda_{p}, p=$ $1,2, \cdots$. Indeed, $\sigma\left(P_{0}\right)=\sigma_{a c}\left(P_{0}\right)=\underset{p \geq 1}{\cup} \Lambda_{p}$. See also $[40]$.

Definition 2.1. Let $\mu \in \mathbb{R}$ and $\mathrm{F}(\mu)=\left\{\mathrm{k} \in \mathbb{T}^{*} ; \mu \in \sigma(\mathrm{P}(\mathrm{k}))\right\}$ the corresponding Fermi-surface. 
a) We will say that $\mu \in \sigma\left(\mathrm{P}_{0}\right)$ is a simple energy level if and only if $\mu$ is a simple eigenvalue of $\mathrm{P}(\mathrm{k})$, for every $\mathrm{k} \in \mathrm{F}(\mu)$.

b) Assume that $\mu$ is a simple energy level of $\mathrm{P}_{0}$ and let $\lambda(\mathrm{k})$ be the unique eigenvalue defined on a neighborhood of $\mathrm{F}(\mu)$ such that $\lambda(\mathrm{k})=\mu$, for all $\mathrm{k} \in \mathrm{F}(\mu)$. We say that $\mu$ is a non-critical energy of $\mathrm{P}_{0}$ if $\mathrm{d}_{\mathrm{k}} \lambda(\mathrm{k}) \neq 0$ for all $\mathrm{k} \in \mathrm{F}(\mu)$.

Note that in one dimension case $F(\mu)$ is just a finite set of points.

Now, let us recall some well-known facts about the density of states associated with $\mathrm{P}_{0}$, see [40]. The density of states measure $\rho$ is defined as follows:

$$
\rho(\mu):=\frac{1}{(2 \pi)^{n}} \sum_{p \geq 1} \int_{\left\{k \in E^{*} ; \lambda_{p}(k) \leq \mu\right\}} d k .
$$

Since the spectrum of $P_{0}$ is absolutely continuous, the measure $\rho$ is absolutely continuous

with respect to the Lebesgue measure $d \mu$. Therefore the density of states, $\frac{d \rho}{d E}(E)$, of $P_{0}$ is locally integrable.

\section{$2.2 \quad$ Results}

We now consider the perturbed periodic Schrödinger operator:

$$
\mathrm{P}(\mathrm{h}):=\mathrm{P}_{0}+\varphi(h x), \quad \mathrm{h} \searrow 0,
$$

where $\varphi \in \mathrm{C}^{\infty}\left(\mathbb{R}^{n} ; \mathbb{R}\right)$ and satisfies:

(A1) There exists $\delta>0$ such that $\forall \alpha \in \mathbb{N}^{n}, \exists C_{\alpha}>0$ s.t.

$$
\left|\partial_{x}^{\alpha} \varphi(x)\right| \leq C_{\alpha}(1+|x|)^{-\delta-|\alpha|} \text { uniformly on } x \in \mathbb{R}^{n} .
$$

The operator $\mathrm{P}(\mathrm{h})$ is self-adjoint, semi-bounded on $\mathrm{L}^{2}\left(\mathbb{R}^{n}\right)$ with domain $\mathrm{H}^{2}\left(\mathbb{R}^{\mathrm{n}}\right)$.

The assumption (A1) and the perturbation theory (Weyl theorem) give:

$$
\sigma_{\text {ess }}(P(h))=\sigma_{\text {ess }}\left(P_{0}\right)=\sigma\left(P_{0}\right)=\bigcup_{p \geq 1} \Lambda_{p} .
$$

Recall that $\sigma_{\text {ess }}(A)$, the essential spectrum of $A$, is defined by $\sigma_{\text {ess }}(A)=\sigma(A) \backslash \sigma_{\text {disc }}(A)$, where $\sigma_{\text {disc }}(A)$ is the set of isolated eigenvalues of $A$ with finite multiplicity. Here $A$ is an unbounded operator on a Hilbert space.

Our first theorem in this section concerns the weak asymptotic of $\zeta_{h}^{\prime}(\mu)$. Let $\left.I=\right] \mathrm{a}, \mathrm{b}[\subset \mathbb{R}$.

Theorem 2.2 (Weak asymptotic). Assume (A1) with $\delta>\mathrm{n}$. For $\mathrm{f} \in \mathcal{C}_{0}^{\infty}(\mathrm{I})$, the operator $\left[\mathrm{f}(\mathrm{P}(\mathrm{h}))-\mathrm{f}\left(\mathrm{P}_{\mathrm{O}}\right)\right]$ is of trace class and

$$
\operatorname{tr}\left[f(P(h))-f\left(P_{0}\right)\right] \sim h^{-n} \sum_{j=0}^{+\infty} a_{j}(f) h^{j}, \quad \text { when } h \searrow 0,
$$


with

$$
a_{0}(f)=(2 \pi)^{-n} \sum_{p \geq 1} \int_{\mathbb{R}_{x}^{n}} \int_{E^{*}}\left[f\left(\lambda_{p}(k)+\varphi(x)\right)-f\left(\lambda_{p}(k)\right)\right] d k d x .
$$

The coefficients $\mathbf{f} \rightarrow \boldsymbol{a}_{\mathbf{j}}(\mathbf{f})$ are distributions of finite order $\leq \mathfrak{j}+1$. Moreover, if $\mu$ is a non-critical energy of $\mathrm{P}_{0}$ for all $\mu \in \mathrm{I}$, then $\boldsymbol{a}_{\mathfrak{j}}(\mathrm{f})=-\left\langle\gamma_{j}(\cdot), f\right\rangle$, for all $\mathrm{f} \in \mathcal{C}_{0}^{\infty}(\mathrm{I})$. Here $\gamma_{j}(\mu)$ are smooth functions of $\mu \in \mathrm{I}$. In particular,

$$
\gamma_{0}(\mu)=\frac{d}{d \mu}\left[\int_{\mathbb{R}_{x}^{n}}\{\rho(\mu)-\rho(\mu-\varphi(x))\} d x\right] .
$$

The proof of Theorem 2.2 is contained in subsection 3.3.

Let $[a, b] \subset \mathbb{R}$. Assume that:

(A2) for all $\mu \in[a, b], \mu$ is a non-critical energy of $P_{0}$.

For all $\mu \in[a, b]$, let $\lambda(k)$ be the unique eigenvalue defined on a neighborhood of $F(\mu)$ such that $\lambda(k)=\mu$. We assume that for all $(k, r) \in \mathbb{T}^{*} \times \mathbb{R}^{n}$ such that $\mu=\lambda(k)+\varphi(r) \in \sigma\left(P_{0}\right) \cap[a, b], \mu$ is a simple energy level, and that:

(A3) $|\nabla \lambda(k)|^{2}-r \nabla \varphi(r) \Delta \lambda(k)>0$, for all $(k, r)$ s.t. $\lambda(k)+\varphi(r) \in[a, b]$.

Remark. Note that the assumption (A2) is fulfilled in the bottom of the spectrum of $\mathrm{P}_{0}$. Moreover, assuming (A2) the hypothesis (A3) is satisfied if $\|\varphi\|_{\infty}+\|x \nabla \varphi\|_{\infty}<<1$, (see [27], [37, 38]).

Our main result concerning the derivative of the spectral shift function is the following.

Theorem 2.3 (Pointwise asymptotic). Assume (A2), (A3) and (A1) with $\delta>\mathrm{n}$. Then the following asymptotic expansion holds:

$$
\zeta_{h}^{\prime}(\mu) \sim h^{-n} \sum_{j \geq 0} \gamma_{j}(\mu) h^{j} \quad \text { as } h \searrow 0
$$

uniformly for $\mu \in[a, b]$.

The coefficients $\left(\gamma_{j}(\mu)\right)_{j \geq 0}$ are given in Theorem 2.2. Furthermore, this expansion has derivate in $\mu$ to any order.

Remark 2.4. Theorems 2.2 and 2.3 still true also in the case when the potential $\varphi(x)$ depend on $\mathrm{h}$, i.e. $\varphi(\mathrm{x}, \mathrm{h})=\varphi(\mathrm{x})+\mathrm{h} \varphi_{1}(\mathrm{x})+\mathrm{h}^{2} \varphi_{2}(\mathrm{x}) \cdots$ in $\mathrm{S}^{\mathcal{\delta}}(1)$. See the next section for the definition of $S^{\mathcal{\delta}}(1)$. (subsection 3.1).

\subsection{Outline of the proofs}

Let $\mathrm{Q}_{1}(\mathrm{~h})=\mathrm{Q}_{1}^{w}\left(x, h \mathrm{D}_{x}\right), \mathrm{Q}_{0}(\mathrm{~h})=\mathrm{Q}_{0}^{w}\left(x, h \mathrm{D}_{\mathrm{x}}\right)$ be two $\mathrm{h}$-pseudodifferential operators such that $\mathrm{Q}_{j}(h)=Q_{j}^{*}(h),(j=0,1)$, and

$$
\left(Q_{1}(h)+i\right)^{-1}-\left(Q_{0}(h)+i\right)^{-1}
$$


is an operator of trace class. In this case, Theorem 2.2 is well-known see [28] and the references therein. On the other hand, in the case of non-trapping geometrie, the asymptotic follows from the results of Robert-Tamura (see [33, 34, 35] and also [31]). The main ingredient in the RobertTamura method is the limiting absorption theorem and the construction of a long-time parametrix for the time-dependent equation

$$
\left(h D_{t}-Q_{j}^{w}\left(x, h D_{x}\right)\right) u_{j}(t)=0, \quad u_{j}(0)=I .
$$

In our case $\mathrm{P}(\mathrm{h})=-\Delta+\mathrm{V}(\mathrm{x})+\varphi(\mathrm{h} x)$ is not an $\mathrm{h}$-pseudodifferential operator. In fact, when $(h \searrow 0)$, there are two spatial scales in equation (1.1). The first one of the order of the linear dimension $\gamma$ of the periodicity cell and the second one of order $\frac{\gamma}{h}$ on which the perturbation of the potential varies appreciably. To remedy this we reduce the study of $\mathrm{P}(\mathrm{h})$, to the one of a system of h-pseudodifferential operators. More precisely, following [7, 9, 12, 15, 18], we can reduce the spectral study of $\mathrm{P}(\mathrm{h})$ near any fixed energy $z$ to the study of a finite system of $h$-pseudodifferential operators, $\mathrm{E}_{-+}(z, \mathrm{~h})$, acting on $\mathrm{L}^{2}\left(\mathbb{T}^{* n} ; \mathbb{C}^{\mathrm{N}}\right)$. In general, for the reduced problem, the dependence on the spectral parameter is non-linear. However, in the case of simple band (see assumption (A2)) we show that

$$
\mathrm{E}_{-+}(z, \mathrm{~h})=z-\left(\lambda(\mathrm{k})+\varphi(\mathrm{r})+\mathrm{hK} \mathrm{K}_{1}(\mathrm{k}, \mathrm{r})+\mathrm{h}^{2} \mathrm{~K}_{2}(\mathrm{k}, \mathrm{r} ; z, \mathrm{~h})\right),
$$

where $\mathrm{K}_{1} \in \mathrm{S}^{\delta+1}\left(\mathbb{T}^{*} \times \mathbb{R}^{\mathrm{n}}\right)$ and $\mathrm{K}_{2}(\cdot ; z, \mathrm{~h}) \in \mathrm{S}^{\delta+2}\left(\mathbb{T}^{*} \times \mathbb{R}^{\mathrm{n}}\right)$, holomorphic with respect to $z$ in a small complex neighborhood $\Omega$ of a bounded interval I. (See 3.24). Now, considering $s \in \Omega \cap \mathbb{R}$ as a parameter and assuming (A3), we can apply the Robert-Tamura approach to the hamiltonian $B_{s}\left(k,-h D_{k} ; h\right):=\lambda(k)+\varphi\left(-h D_{k}\right)+h K_{1}^{w}\left(k,-h D_{k}\right)+h^{2} G^{w}\left(k,-h D_{k} ; s, h\right)$ where G satisfies the same properties as $\mathrm{K}_{2}$, and we obtain the theorem 2.3. Here we use the following crucial argument: the assumption (A3) implies that the interval $\mathrm{I}$ is a non-trapping region of the classical hamiltonian associated to $B_{s}$ for all $s$ in the compact set $\Omega \cap \mathbb{R}$. In fact, $r \cdot K_{i}(k, r) \in S^{\delta}\left(\mathbb{T}^{*} \times \mathbb{R}^{n}\right) \subset S^{\mathcal{O}}\left(\mathbb{T}^{*} \times \mathbb{R}^{n}\right)$, $(i=1,2)$ then the corresponding operators are bounded uniformly for $s \in \Omega \cap \mathbb{R}$ and moreover, the principal symbol of $B_{s}$ does not depend on $s$.

\section{Proofs}

\subsection{Definitions and notations}

Let $\mathrm{H}$ be a Hilbert space. The scalar product in $\mathrm{H}$ will be denoted by $\langle\cdot, \cdot\rangle$. The set of linear bounded operators from $\mathrm{H}_{1}$ to $\mathrm{H}_{2}$ is denoted by $\mathcal{L}\left(\mathrm{H}_{1}, \mathrm{H}_{2}\right)$.

For $(m, N) \in \mathbb{R} \times \mathbb{N}$ we denote by $S^{m}\left(\mathbb{T}^{*} \times \mathbb{R}^{n} ; \mathcal{M}_{N}(\mathbb{C})\right)$ the space of $\mathrm{P} \in \mathrm{C}^{\infty}\left(\mathbb{R}_{\mathrm{k}, \mathrm{r}}^{2 \mathrm{n}} ; \mathcal{M}_{\mathrm{N}}(\mathbb{C})\right)$, $\Gamma^{*}$-periodic with respect to $k$, such that for all $\alpha$ and $\beta$ in $\mathbb{N}^{n}$ there exists $C_{\alpha, \beta}>0$ such that

$$
\left\|\partial_{r}^{\alpha} \partial_{k}^{\beta} \mathrm{P}(k, r)\right\|_{\mathcal{M}_{N}(\mathbb{C})} \leq C_{\alpha, \beta}\langle r\rangle^{-m-|\alpha|}, \quad\langle r\rangle=\left(1+|r|^{2}\right)^{\frac{1}{2}},
$$

where $\mathcal{M}_{N}(\mathbb{C})$ is the set of $\mathrm{N} \times \mathrm{N}$-matrices. In the special case when $\mathrm{N}=1$ (i.e., $\mathrm{P}$ is real valued), we will write $S^{\mathfrak{m}}\left(\mathbb{T}^{*} \times \mathbb{R}^{n}\right)$ instead of $S^{\mathfrak{m}}\left(\mathbb{T}^{*} \times \mathbb{R}^{n} ; \mathcal{M}_{1}(\mathbb{C})\right)$. 
If $P$ depends on a semi-classical parameter $\left.h \in] 0, h_{0}\right]$ and possibly on other parameters as well, we require (3.1) to hold uniformly with respect to these parameters. For $h$ dependent symbols, we say that $\mathrm{P}(\mathrm{k}, \mathrm{r} ; \mathrm{h})$ has an asymptotic expansion in powers of $h$, and we write

$$
P(k, r ; h) \sim \sum_{j=0}^{\infty} P_{j}(k, r) h^{j},
$$

if for every $N \in \mathbb{N}, h^{-(N+1)}\left(P-\sum_{j=0}^{N} P_{j} h^{j}\right) \in S^{m}\left(T^{*} \times \mathbb{R}^{n} ; \mathcal{M}_{N}(\mathbb{C})\right)$.

For $\mathrm{P} \in \mathrm{S}^{\mathrm{m}}\left(\mathbb{T}^{*} \times \mathbb{R}^{\mathrm{n}} ; \mathcal{M}_{\mathrm{N}}(\mathbb{C})\right)$, the $h$-Weyl operator $\mathrm{P}=\mathrm{P}^{w}\left(\mathrm{k}, \mathrm{hD}_{\mathrm{k}} ; h\right)=\mathrm{Op} \mathrm{p}_{\mathrm{h}}^{w}(\mathrm{P})$ is defined by:

$$
P^{w}\left(k, h D_{k} ; h\right) u(k)=(2 \pi h)^{-n} \iint e^{\frac{i}{h}(k-y) r} P\left(\frac{k+y}{2}, r ; h\right) u(y) d y d r . \text { Here } D_{k}=\frac{1}{i} \frac{\partial}{\partial k} .
$$

\subsection{Effective Hamiltonian}

In this subsection, we recall the effective Hamiltonian method. More precisely, we will construct a suitable auxiliary (so-called Grushin) problem associated with the operator $(\mathrm{P}(\mathrm{h})-z)$ for $z$ in a small complex neighborhood of $I$, where $I=[a, b] \subset \mathbb{R}$ is some bounded interval. The reader can find more details and the proofs of the results of this subsection in [20] (see also [11, 12, 15]). For the reader convenience, let us point out the main change in our situation and fix the notations.

Denote by $T_{\Gamma}$ the distribution in $\mathcal{S}^{\prime}\left(\mathbb{R}^{2 n}\right)$ defined by $T_{\Gamma}(x, y)=\frac{1}{\operatorname{vol}(E) h^{n}} \sum_{\beta^{*} \in \Gamma^{*}} e^{i(x-h y) \frac{\beta^{*}}{h}}$. We recall that $E$ is a fundamental domain of $\Gamma$.

For $\mathrm{m} \in \mathbb{N}$, put $\mathbb{L}^{\mathrm{m}}:=\left\{\mathrm{u}(\mathrm{x}) \mathrm{T}_{\Gamma}(x, y) ; \partial_{\chi}^{\alpha} \mathrm{u} \in \mathrm{L}^{2}\left(\mathbb{R}^{\mathrm{n}}\right), \forall \alpha,|\alpha| \leq \mathrm{m}\right\}$.

It was shown in $[11$, Chapter 13 , Proposition 13.5$]$, that the operator $\mathrm{P}(\mathrm{h})$ acting on $\mathrm{L}^{2}\left(\mathbb{R}^{\mathrm{n}}\right)$ with domain $\mathrm{H}^{2}\left(\mathbb{R}^{n}\right)$ is unitary equivalent to

$$
\mathbb{P}_{1}(h):=\left(D_{y}+h D_{x}\right)^{2}+V(y)+\varphi(x),
$$

acting on $\mathbb{L}^{0}$ with domain $\mathbb{L}^{2}$, and the following proposition holds.

Proposition 3.1. Assume (A1). There exist $\mathrm{N} \in \mathbb{N}$, a complex neighborhood $\Omega$ of $\mathrm{I}$, and a bounded operator $\mathrm{R}_{+}$in $\mathcal{L}\left(\mathbb{L}^{0} ; \mathrm{L}^{2}\left(\mathbb{T}^{*} ; \mathbb{C}^{\mathrm{N}}\right)\right.$ ) such that for all $z \in \Omega$ and $0<\mathrm{h}<\mathrm{h}_{0}$ small enough, the operator

$$
\mathcal{P}_{1}(z, h):=\left(\begin{array}{cc}
\mathbb{P}_{1}(h)-z & \mathrm{R}_{+}^{*} \\
\mathrm{R}_{+} & 0
\end{array}\right): \mathbb{L}^{2} \times \mathrm{L}^{2}\left(\mathbb{T}^{*} ; \mathbb{C}^{\mathrm{N}}\right) \rightarrow \mathbb{L}^{0} \times \mathrm{L}^{2}\left(\mathbb{T}^{*} ; \mathbb{C}^{\mathrm{N}}\right)
$$

is bijective with bounded two-sided inverse

$$
\mathcal{E}_{1}(z, h):=\left(\begin{array}{cc}
E_{1}(z, h) & E_{1,+}(z, h) \\
E_{1,-}(z, h) & E_{1,-+}(z, h)
\end{array}\right) .
$$


Here $\mathrm{E}_{1,-+}:=\mathrm{E}_{1,-+}^{w}\left(\mathrm{k},-\mathrm{hD}_{\mathrm{k}} ; \mathrm{z}, \mathrm{h}\right)$ is an $\mathrm{h}-$ pseudodifferential operator with symbol

$$
\mathrm{E}_{1,-+}(\mathrm{k}, \mathrm{r} ; z, \mathrm{~h}) \sim \sum_{\mathrm{l} \geq 0} \mathrm{E}_{1,-+}^{\mathrm{l}}(\mathrm{k}, \mathrm{r} ; z) \mathrm{h}^{\mathrm{l}}, \quad \forall 0<\mathrm{h}<\mathrm{h}_{0},
$$

in $\mathrm{S}^{\mathrm{O}}\left(\mathbb{T}^{*} \times \mathbb{R}^{\mathrm{n}} ; \mathcal{L}\left(\mathbb{C}^{\mathrm{N}}, \mathbb{C}^{\mathrm{N}}\right)\right)$

Remark 3.2. (1) We denote by $\mathcal{P}_{0}(z, h)$ and $\mathcal{E}_{0}(z, h):=\left(\begin{array}{cc}E_{0}(z, h) & E_{0,+}(z, h) \\ E_{0,-}(z, h) & E_{0,-+}(z, h)\end{array}\right)$ the operators given by Proposition 3.1 when $\varphi=0$.

(2) Note that, $\mathrm{R}_{+}$depends only on the non-perturbed periodic Schrödinger operator $\mathrm{P}_{0}$. See [15, Proposition 2.1] and [11, Chapter 13]. Therefore, we may take the same $\mathrm{R}_{+}$for $\mathcal{P}_{1}(z, \mathrm{~h})$ and $\mathcal{P}_{0}(z, h)$.

The following well-known formulas are a consequence of Proposition 3.1 (see also [20]), for $j=0,1$.

$$
\begin{gathered}
\left(\mathbb{P}_{j}(h)-z\right)^{-1}=E_{j}(z, h)-E_{j,+}(z, h) E_{j,-+}(z, h)^{-1} E_{j,-}(z, h), \\
E_{j,-+}(z, h)^{-1}=-R_{+}\left(\mathbb{P}_{j}(h)-z\right)^{-1} R_{+}^{*}
\end{gathered}
$$

and

$$
\partial_{z} E_{j,-+}(z, h)=E_{j,-}(z, h) E_{j,+}(z, h) .
$$

Here $\mathbb{P}_{0}(h):=\left(D_{y}+h D_{x}\right)^{2}+V(y)$.

We observe that $\mathcal{P}_{\mathrm{j}}(z, \mathrm{~h})^{*}=\mathcal{P}_{\mathrm{j}}(\bar{z}, \mathrm{~h})$, which implies that $\mathcal{E}_{\mathrm{j}}(z, \mathrm{~h})^{*}=\mathcal{E}_{\mathbf{j}}(\bar{z}, \mathrm{~h})$. From this, we deduce the following identity:

$$
E_{j,-+}(z, h)^{*}=E_{j,-+}(\bar{z}, h), \quad j=0,1 .
$$

In the following, we write $\left[a_{j}\right]_{j=0}^{1}=a_{1}-a_{0}$.

Lemma 3.3. We have

$$
\begin{aligned}
& {\left[E_{j,+}(z, h)\right]_{j=0}^{1}=E_{1}(z, h) \varphi(r) E_{0,+}(z, h),} \\
& {\left[E_{j,-}(z, h)\right]_{j=0}^{1}=E_{0,-}(z, h) \varphi(r) E_{1}(z, h),}
\end{aligned}
$$

and

$$
\left[\mathrm{E}_{j,-+}(z, h)\right]_{j=0}^{1}=E_{1,-}(z, h) \varphi(r) E_{0,+}(z, h) .
$$

In particular, if (A1) is satisfied then

$$
\left[E_{j,-+}(k, r ; z, h)\right]_{j=0}^{1} \in S^{\delta}\left(\mathbb{T}_{k}^{*} \times \mathbb{R}_{r}^{n} ; \mathcal{M}_{N}\left(\mathbb{C}^{N}\right)\right) .
$$


Proof. Identities (3.10)-(3.12) follow from the first resolvent equation

$$
\begin{aligned}
{\left[\mathcal{E}_{j}(z, h)\right]_{j=0}^{1} } & =\mathcal{E}_{1}(z, h)\left[\mathcal{P}_{0}(z, h)-\mathcal{P}_{1}(z, h)\right] \mathcal{E}_{0}(z, h) \\
& =-\mathcal{E}_{0}(z, h)\left[\mathcal{P}_{1}(z, h)-\mathcal{P}_{0}(z, h)\right] \mathcal{E}_{1}(z, h)
\end{aligned}
$$

and the fact that $\left[\mathcal{P}_{j}(z, h)\right]_{j=0}^{1}=\left(\begin{array}{cc}\varphi(r) & 0 \\ 0 & 0\end{array}\right)$.

Formula (3.13) is a simple consequence of (3.12) and standard h-pseudodifferential calculus.

Lemma 3.4. Assume (A1) with $\delta>n$, the operator

$$
\varphi(r) \mathrm{E}_{0,+}(z, h): \mathrm{L}^{2}\left(\mathbb{T}^{*} ; \mathbb{C}^{\mathrm{N}}\right) \rightarrow \mathrm{L}^{2}\left(\mathbb{R}^{\mathrm{n}}\right),
$$

and

$$
E_{0,-}(z, h) \varphi(r): L^{2}\left(\mathbb{R}^{n}\right) \rightarrow L^{2}\left(\mathbb{T}^{*} ; \mathbb{C}^{N}\right)
$$

are of trace class.

Proof. Since $\left(\mathrm{E}_{0,-}(z, \mathrm{~h}) \varphi(\mathrm{r})\right)^{*}=\varphi(\mathrm{r}) \mathrm{E}_{0,+}(\bar{z}, \mathrm{~h})$ it suffice to prove 3.14. Without any loss of generality, we may assume that $\mathrm{N}=1$.

Consider the operator $A=\left(\mathrm{Id}-\mathrm{h}^{2} \Delta_{\mathbb{T}^{*}}\right)^{-\frac{\delta}{2}}$ on $\mathrm{L}^{2}\left(\mathbb{T}^{*} ; \mathbb{C}\right)$. Set $B=\varphi(r) E_{0,+}(z, h), C=B^{*} B$ and $\mathrm{D}=\mathrm{A}^{-1} \mathrm{CA}-1$.

Since $\varphi \in S^{\delta}\left(\mathbb{R}^{2 n}\right)$ and $E_{0,+}(k, r ; z, h) \in S^{0}$, a standard result of $h$-pseudodifferential calculus shows that $\mathrm{D} \in \mathrm{S}^{0}\left(\mathbb{T}_{\mathrm{k}}^{*} \times \mathbb{R}_{\mathrm{r}}^{\mathrm{n}}\right)$. Therefore, D extends to a bounded operator from $\mathrm{L}^{2}\left(\mathbb{T}^{*} ; \mathbb{C}\right)$ into $\mathrm{L}^{2}\left(\mathbb{T}^{*} ; \mathbb{C}\right)$, see $[11$, Chapter 13$]$. Combining this with the fact that $\mathrm{C}$ is positif, we get:

$$
0 \leq \mathrm{C}=\mathrm{ADA} \leq\|\mathrm{D}\| A^{2}
$$

which implies

$$
0 \leq C^{\frac{1}{2}} \leq \sqrt{\|\mathrm{D}\|} A
$$

Since $\delta>n$ then $A: L^{2}\left(\mathbb{T}^{*} ; \mathbb{C}\right) \rightarrow \mathrm{L}^{2}\left(\mathbb{T}^{*} ; \mathbb{C}\right)$ is of trace class and the lemma follows from the above inequality.

Remark 3.5. Notice that if $\mathrm{P} \in \mathrm{S}^{\delta}\left(\mathbb{T}^{*} \times \mathbb{R}^{\mathrm{n}} ; \mathcal{M}_{\mathrm{N}}(\mathbb{C})\right)$ with $\delta>\mathrm{n}$, then the operator $\mathrm{P}^{w}\left(\mathrm{k}, \mathrm{hD} \mathrm{b}_{\mathrm{k}}\right)$ is a trace class, see [11].

Proposition 3.6. Assume (A1) with $\delta>n$. For $z \in \Omega$ such that $\mathfrak{I}(z) \neq 0$, the operator

$$
\left[E_{j,+}(z, h) E_{j,-+}(z, h)^{-1} E_{j,-}(z, h)\right]_{j=0}^{1}
$$


is of trace class from $\mathrm{L}^{2}\left(\mathbb{R}^{\mathrm{n}}\right)$ to $\mathrm{L}^{2}\left(\mathbb{R}^{\mathrm{n}}\right)$ and

$$
\operatorname{tr}\left(\left[E_{j,+}(z, h) E_{j,-+}(z, h)^{-1} E_{j,-}(z, h)\right]_{j=0}^{1}\right)=\operatorname{tr}\left(\left[E_{j,-+}(z, h)^{-1} \partial_{z} E_{j,-+}(z, h)\right]_{j=0}^{1}\right) .
$$

Here the operator in the right member of $(3.16)$ is defined on $\mathrm{L}^{2}\left(\mathbb{T}^{*} ; \mathbb{C}^{\mathrm{N}}\right)$.

Proof. Let $z \in \Omega$ such that $\mathfrak{I}(z) \neq 0$, we have the following identity:

$$
\begin{aligned}
& {\left[E_{j,+}(z, h) E_{j,-+}(z, h)^{-1} E_{j,-}(z, h)\right]_{j=0}^{1}=} \\
& {\left[\left(\left[E_{j,+}(z, h)\right]_{j=0}^{1}\right) E_{1,-+}(z, h)^{-1} E_{1,-}(z, h)\right]+} \\
& {\left[E_{0,+}(z, h) E_{0,-+}(z, h)^{-1}\left(\left[E_{j,-}(z, h)\right]_{j=0}^{1}\right)\right]-} \\
& {\left[E_{0,+}(z, h) E_{1,-+}(z, h)^{-1}\left(\left[E_{j,-+}(z, h)\right]_{j=0}^{1}\right) E_{0,-+}(z, h)^{-1} E_{1,-}(z, h)\right] .}
\end{aligned}
$$

According to Lemmas 3.3 and 3.4, all the term of the right member in the last equality are of trace class. Using the cyclicity of the trace and identity (3.8), we obtain the proposition.

Using again the cyclicity of the trace in (3.17) and the identity (3.8) we obtain

$$
\operatorname{tr}\left(\left[E_{j,-+}(z, h)^{-1} \partial_{z} E_{j,-+}(z, h)\right]_{j=0}^{1}\right)=\operatorname{tr}\left(\left[\partial_{z} E_{j,-+}(z, h) E_{j,-+}(z, h)^{-1}\right]_{j=0}^{1}\right) .
$$

The main result in this subsection is

Proposition 3.7. Assume (A1) with $\delta>n$. Let $\psi \in \mathcal{C}_{0}^{\infty}(\mathbb{R})$ and let $\widetilde{\psi}$ be an almost analytic extension of $\psi$. Then the operator $\left[\psi(\mathrm{P}(\mathrm{h}))-\psi\left(\mathrm{P}_{0}\right)\right]$ is of trace class as an operator from $\mathrm{L}^{2}\left(\mathbb{R}^{\mathrm{n}}\right)$ to $\mathrm{L}^{2}\left(\mathbb{R}^{\mathrm{n}}\right)$ and

$$
\begin{aligned}
\operatorname{tr}\left[\psi(P(h))-\psi\left(P_{0}\right)\right]=\operatorname{tr}\left[\psi\left(\mathbb{P}_{1}(h)\right)-\right. & \left.\psi\left(\mathbb{P}_{0}(h)\right)\right]= \\
& -\frac{1}{\pi} \int_{\mathbb{C}} \bar{\partial} \widetilde{\psi}(z) \operatorname{tr}\left(\left[E_{j,-+}(z)^{-1} \partial_{z} E_{j,-+}\right]_{j=0}^{1}\right) L(d z) .
\end{aligned}
$$

Here $\bar{\partial}=\frac{\partial}{\partial \bar{z}}$ and $\mathrm{L}(\mathrm{d} z)=\mathrm{d} x \mathrm{dy}$ denotes the Lebesgue measure on $\mathbb{C}$.

Recall that $\widetilde{\psi} \in \mathcal{C}_{0}^{\infty}(\mathbb{C})$ is an almost analytic extension of $\psi$, i.e. $\widetilde{\psi}_{\mid \mathbb{R}}=\psi$ and $\bar{\partial} \widetilde{\psi}=\mathcal{O}\left(|\mathfrak{I}(z)|^{N}\right)$ for all $N \in \mathbb{N}$. We refer to [11] for the existence of $\widetilde{\psi}$.

Proof. By Helffer-Sjöstrand formula (see [19]), we have

$$
\psi\left(\mathbb{P}_{1}(h)\right)-\psi\left(\mathbb{P}_{0}(h)\right)=-\frac{1}{\pi} \int_{\mathbb{C}} \bar{\partial} \widetilde{\psi}(z)\left[\left(z-\mathbb{P}_{1}(h)\right)^{-1}-\left(z-\mathbb{P}_{0}(h)\right)^{-1}\right] \mathrm{L}(\mathrm{d} z) .
$$


Combining this with (3.6), we obtain

$$
\begin{aligned}
\psi\left(\mathbb{P}_{1}(h)\right)-\psi\left(\mathbb{P}_{0}(h)\right)= & \frac{1}{\pi} \int_{\mathbb{C}} \bar{\partial} \widetilde{\psi}(z)\left[E_{j}(z, h)\right]_{j=0}^{1} L(d z) \\
& -\frac{1}{\pi} \int_{\mathbb{C}} \bar{\partial} \widetilde{\psi}(z)\left[E_{j,+}(z, h) E_{j,-+}(z, h)^{-1} E_{j,-}(z, h)\right]_{j=0}^{1} L(d z) .
\end{aligned}
$$

Since $E_{j}(z, h), j=0,1$ is holomorphic in a neighborhood of $\operatorname{supp}(\widetilde{\psi})$, the first term in the right member of (3.20) vanishes. Consequently,

$$
\psi\left(\mathbb{P}_{1}(h)\right)-\psi\left(\mathbb{P}_{0}(h)\right)=-\frac{1}{\pi} \int_{\mathbb{C}} \bar{\partial} \widetilde{\psi}(z)\left[E_{j,+}(z, h) E_{j,-+}(z, h)^{-1} E_{j,-}(z, h)\right]_{j=0}^{1} L(d z) .
$$

Using Proposition 3.6, we conclude that $\left[\psi\left(\mathbb{P}_{1}(h)\right)-\psi\left(\mathbb{P}_{0}(h)\right)\right]$ is of trace class and applying (3.16), we obtain the second equality of (3.19). The first equality follows from the fact that $\mathbb{P}_{1}(\mathrm{~h})$ (resp. $\mathbb{P}_{0}(h)$ ) is unitary equivalent to $\mathrm{P}(\mathrm{h})$ (resp. $\mathrm{P}_{0}$ ).

Now, we recall a representation of the derivative of the spectral shift function in term of the effective Hamiltonian $\mathrm{E}_{\mathbf{j},-+}(\boldsymbol{z}, \mathrm{h})$ (see [10, Lemma 1]).

Let $\mathrm{I} \subset \mathbb{R}$ be some bounded interval and $\Omega$ be the complex neighborhood of I given by the proposition 3.1. Put $\Omega_{ \pm}=\Omega \cap\{z \in \mathbb{C} ; \pm \mathfrak{I}(z)>0\}$.

We introduce the functions $e_{ \pm}(z)=\operatorname{tr}\left(\left[\mathrm{E}_{j,-+}(z, h)^{-1} \partial_{z} E_{j,-+}(z, h)\right]_{j=0}^{1}\right)$, for $z \in \Omega_{ \pm}$. Since $E_{j,-+}(z, h)$ is holomorphic on $z$, we deduce from (3.9) that

$$
\partial_{z} E_{j,-+}(z, h)^{*}=\partial_{z} E_{j,-+}(\bar{z}, h) .
$$

Using the fact that $\overline{\operatorname{tr}(A)}=\operatorname{tr}\left(A^{*}\right)$ with (3.9), (3.18) and the above equality we obtain

$$
\overline{e_{+}(z)}=e_{-}(\bar{z}), \quad \text { for all } z \in \Omega_{+} .
$$

Lemma 3.8. [10, Lemma 1] Assume (A1) with $\delta>n$. In $\mathcal{D}^{\prime}(\mathrm{I})$, we have

$$
\zeta_{h}^{\prime}(\mu)=\lim _{\epsilon \searrow 0} \frac{1}{2 \pi i}\left[e_{+}(\mu+i \epsilon)-\overline{e_{+}(\mu+i \epsilon)}\right] .
$$

For the reader convenience we give the proof of the lemma.

Proof. Let $\psi \in \mathcal{C}_{0}^{\infty}(\mathrm{I})$. In the previous proposition, we proved that

$$
-\left\langle\zeta_{h}^{\prime}(\cdot), \psi\right\rangle=\operatorname{tr}\left[\psi(P(h))-\psi\left(P_{0}\right)\right]=-\frac{1}{\pi} \int_{\mathbb{C}} \bar{\partial} \widetilde{\psi}(z) \operatorname{tr}\left(\left[E_{j,-+}(z)^{-1} \partial_{z} E_{j,-+}\right]_{j=0}^{1}\right) L(d z) .
$$

Since $e_{ \pm}(z)=\mathcal{O}\left(h^{-\mathfrak{n}}|\Im(z)|^{-2}\right)$ and $\bar{\partial} \widetilde{\psi}(z)=\mathcal{O}\left(|\Im(z)|^{2}\right)$, the integral in the identity above converge. Thus the r.h.s. of the previous equality can be written as

$$
-\left\langle\zeta_{h}^{\prime}(\cdot), \psi\right\rangle=\lim _{\epsilon \searrow 0}-\frac{1}{\pi}\left[\int_{\mathfrak{I}(z)>0} \bar{\partial} \widetilde{\psi}(z) e_{+}(z+i \epsilon) L(d z)+\int_{\mathfrak{I}(z)<0} \bar{\partial} \widetilde{\psi}(z) e_{-}(z-i \epsilon) L(d z)\right] .
$$


Since $e_{ \pm}(z \pm i \epsilon)$ is holomorphic in $\Omega_{ \pm}$, Green's formula then gives

$$
-\left\langle\zeta_{h}^{\prime}(\cdot), \psi\right\rangle=\lim _{\epsilon \searrow 0} \frac{i}{2 \pi} \int_{\mathbb{R}} \psi(\mu)\left[e_{+}(\mu+i \epsilon)-e_{-}(\mu-i \epsilon)\right] d \mu .
$$

Thus Lemma 3.8 follows from (3.21) and (3.23).

In the following we will use the result concerning the expression of the effective hamiltonian $E_{j,-+}(z, h), j=0,1$ given in [7] (see also [10]). In fact, under the assumptions (A1)-(A2), the two leading terms of the symbol $E_{1,-+}(k, r ; z, h)$ are computed in $[7$, section 4 , formulas (4.5)-(4.7)], it was shown that:

$$
\mathrm{E}_{1,-+}(\mathrm{k}, \mathrm{r} ; z, \mathrm{~h})=z-\left(\lambda(\mathrm{k})+\varphi(\mathrm{r})+\mathrm{h} \mathrm{K}_{1}(\mathrm{k}, \mathrm{r})+\mathrm{h}^{2} \mathrm{~K}_{2}(\mathrm{k}, \mathrm{r} ; z, \mathrm{~h})\right),
$$

where $K_{1} \in S^{\delta+1}\left(\mathbb{T}^{*} \times \mathbb{R}^{n}\right)$ and $K_{2}(\cdot ; z, h) \in S^{\delta+2}\left(\mathbb{T}^{*} \times \mathbb{R}^{n}\right)$, holomorphic with respect to $z$ in $\Omega$. Note that

$$
\mathrm{E}_{0,-+}(\mathrm{k}, \mathrm{r} ; z)=z-\lambda(\mathrm{k}), \quad \mathrm{k} \in \mathbb{T}^{*}, z \in \Omega \text {. }
$$

From now on, we consider the $h$-pseudodifferential operator $\mathrm{H}^{w}\left(k,-h \mathrm{D}_{k} ; \mathrm{h}\right)$ with the following symbol:

$$
H(k, r ; h)=\lambda(k)+\varphi(r)+h K_{1}(k, r) .
$$

Remark that this operator is $z$-independent.

Corollary 3.9. Under Assumptions (A1) with $\delta>n$ and (A2), there exists $\mathrm{G}(\mathrm{k}, \mathrm{r} ; z, \mathrm{~h}) \sim$ $\sum_{j=0}^{\infty} g_{j}(k, r ; z) h^{j}$ in $\mathrm{S}^{\delta+2}\left(\mathbb{T}^{*} \times \mathbb{R}^{n}\right)$ such that for $\mu \in \mathrm{I}$ and $\mathrm{h}$ small enough, we have:

$$
\zeta_{h}^{\prime}(\mu)=\lim _{\epsilon \searrow 0} \frac{1}{2 \pi i}\left[\operatorname{tr}\left(\left(z-B_{\mu}\right)^{-1}-(z-\lambda(k))^{-1}\right)\right]_{z=\mu-i \epsilon}^{z=\mu+i \epsilon}
$$

where $\mathrm{B}_{\mu}:=\mathrm{H}^{w}\left(\mathrm{k},-\mathrm{hD} \mathrm{D}_{\mathrm{k}} ; \mathrm{h}\right)+\mathrm{h}^{2} \mathrm{G}^{w}(\mathrm{k},-\mathrm{hD}, \mu ; \mathrm{h})$. Here $\mathrm{H}(\mathrm{k}, \mathrm{r} ; \mathrm{h})$ is given by 3.26.

Proof. Identity (3.24) gives $\partial_{z} \mathrm{E}_{1,-+}(\mathrm{k}, \mathrm{r} ; z, \mathrm{~h})=1+\mathrm{h}^{2} \partial_{z} \mathrm{~K}_{2}(\mathrm{k}, \mathrm{r} ; z, \mathrm{~h})$ and since $\partial_{z} \mathrm{~K}_{2} \in \mathrm{S}^{\delta+2}\left(\mathbb{T}^{*} \times\right.$ $\left.\mathbb{R}^{n}\right) \subset S^{0}\left(\mathbb{T}^{*} \times \mathbb{R}^{n}\right)$ it follows from the Calderon-Vaillancourt's theorem and the Beal's characterization (see [11]) that the corresponding operator $\partial_{z} E_{1,-+}(z, h)$ is invertible for $h$ small enough and his inverse is given by

$$
\left[\partial_{z} \mathrm{E}_{1,-+}(z)\right]^{-1}=\mathrm{I}+\mathrm{h}^{2} \mathcal{R}(z)
$$

where $\mathcal{R}(z)$ is an $h$-pseudodifferential operator with symbol satisfying the same properties as $\mathrm{K}_{2}$. Combining this with (3.24) and using the composition formula of $h$-pseudodifferential operators we see that there exists $G \sim \sum_{j=0}^{\infty} g_{j}(k, r ; z) h^{j}$ in $S^{\delta+2}\left(\mathbb{T}^{*} \times \mathbb{R}^{n}\right)$ such that

$$
\left(\partial_{z} E_{1,-+}(z, h)\right)^{-1} E_{1,-+}(z, h)=z-H^{w}\left(k,-h D_{k} ; h\right)+h^{2} G^{w}\left(k,-h D_{k} ; z, h\right),
$$

which together with (3.25), Lemma 3.8 and the holomorphy of $\mathrm{G}$ on $z$ give the corollary. 


\subsection{Proof of the weak asymptotic expansion of $\xi_{h}^{\prime}(\cdot)$}

Let $\mathrm{I}=] \mathrm{a}, \mathrm{b}\left[\in \mathbb{R}\right.$ and $\mathrm{f} \in \mathcal{C}_{0}^{\infty}(\mathrm{I})$. The proof of Theorem 2.2 is a simple consequence of Proposition 3.7 (with $\psi=f$ ) and symbolic calculus. Here, we only give an outline of the proof. For the details, we refer to [7]. Fix $\epsilon$ in $] 0, \frac{1}{2}\left[\right.$. The integral (3.19) over $\left\{z \in \mathbb{C} ;|\Im z| \leq h^{\epsilon}\right\}$ is $\mathcal{O}\left(h^{\infty}\right)$, since $\bar{\partial} \widetilde{f}(z)=\mathcal{O}\left(|\Im z|^{\infty}\right)$ and

$$
\left\|\left[\mathrm{E}_{j,-+}(z)^{-1} \partial_{z} \mathrm{E}_{\mathfrak{j},-+}(z)\right]_{j=0}^{1}\right\|_{\operatorname{tr}}=\mathcal{O}\left(|\mathfrak{I} z|^{-1}\right) .
$$

On the other hand, $\left[E_{j,-+}(z, h)^{-1} \partial_{z} E_{j,-+}(z, h)\right]_{j=0}^{1}$ has an asymptotic expansion in powers of $h$ uniformly for $z$ in $\left\{z \in \operatorname{supp} \tilde{f} ;|\Im(z)| \geq h^{\epsilon}\right\}$ (see [7]). Therefore, as in [11, Theorem 13.28], we have

$$
-\frac{1}{\pi} \int_{\mathbb{C}} \tilde{\partial} \widetilde{f}(z) \operatorname{tr}\left(\left[E_{j,-+}(z)^{-1} \partial_{z} E_{j,-+}(z)\right]_{j=0}^{1}\right) L(d z) \sim h^{-n} \sum_{j \geq 0} a_{j} h^{j},(h \searrow 0)
$$

with

$$
a_{0}=(2 \pi)^{-n} \sum_{p \geq 1} \int_{\mathbb{R}_{x}^{n}}\left(\int_{E^{*}}\left[f\left(\lambda_{p}(k)+\varphi(x)\right)-f\left(\lambda_{p}(k)\right)\right] d k\right) d x .
$$

Note that, the sum in the last equality is finite, since $\lim _{p \rightarrow+\infty} \lambda_{p}(k)=+\infty$ and $\varphi$ is bounded.

The coefficient $a_{j}$ is a finite sum of term of the form $\iint c_{l}(x, k) f^{(l)}(b(x, k)) d x d k$, where $c_{l}$ depends on $\varphi$ and their derivatives and $b(x, k) \in\left\{\lambda_{p}(k), \lambda_{p}(k)+\varphi(x)\right\}$, which complete the first part of the theorem 2.2. See [11, Chapter 8, Identity (8.16)].

If $\mu$ is a non-critical energy of $P_{0}$ for all $\mu \in I$. Then $d\left(\lambda_{p}(k)\right) \neq 0$ and $d\left(\lambda_{p}(k)+\varphi(x)\right) \neq 0$ for all $k \in F(\mu)$. We recall that $F(\mu)$ is the Fermi surface. Therefore, $a_{j}(f)=-\left\langle\gamma_{j}(\cdot), f\right\rangle$, for all $f \in$ $\mathcal{C}_{0}^{\infty}(\mathrm{I})$ and $\gamma_{j}(\mu)$ are smooth functions of $\mu \in \mathrm{I}$, in particular, $\gamma_{0}(\mu)=\frac{d}{d \mu}\left[\int_{\mathbb{R}_{x}^{n}} \rho(\mu)-\rho(\mu-\varphi(x)) d x\right]$, which complete the proof of the theorem 2.2.

\subsection{Limiting absorption Theorem}

In this subsection we establish a limiting absorption principle for the operator $\mathrm{P}(\mathrm{h})$, see Theorem 3.11 below. We start by the following lemma, let $[a, b] \in \mathbb{R}$ and $\Omega$ given by Proposition 3.1.

Lemma 3.10. Assume that (A1), (A2) and (A3) are satisfied on $[\mathrm{a}, \mathrm{b}]$. Then, for $\mathrm{l} \in \mathbb{N}^{*}$, there exists $\mathrm{h}_{\mathrm{O}}(\mathrm{l})>0$ small enough such that for all $\mathrm{O}<\mathrm{h}<\mathrm{h}_{\mathrm{O}}(\mathrm{l})$ :

$$
\left\|<h D_{k}>^{-\alpha} E_{j,-+}\left(k,-h D_{k}, \mu \pm i 0 ; h\right)^{-l}<h D_{k}>^{-\alpha}\right\|=\mathcal{O}\left(h^{-l}\right), \quad(j=0,1),
$$

for all $\alpha>l-\frac{1}{2}$ and uniformly for $\mu \in[\mathrm{a}, \mathrm{b}]$, where $\|\cdot\|$ denotes the operator norm when considered as an operator from $\mathrm{L}^{2}\left(\mathbb{T}_{\mathrm{k}}^{*}\right)$ into itself.

Proof. Recall that $\Omega$ is a small complex neighborhood of $[a, b]$ such that the proposition 3.1 holds. For $s \in \Omega \cap \mathbb{R}$, we consider the $h$-pseudodifferential operator $\widetilde{\mathrm{P}}_{s}\left(k,-h D_{k} ; h\right):=H^{w}\left(k,-h D_{k} ; h\right)+$ 
$h^{2} K_{2}^{w}\left(\left(k,-h D_{k} ; s, h\right)\right.$ with the following symbol:

$$
\widetilde{\mathrm{P}}_{\mathrm{s}}(k, r ; h)=\mathrm{H}(k, r ; h)+h^{2} K_{2}(k, r ; s, h),
$$

where $\mathrm{H}(\mathrm{k}, \mathrm{r} ; \mathrm{h})$ given by $(3.26)$ and $\mathrm{K}_{2}(\cdot ; z, h) \in \mathrm{S}^{\delta+2}\left(\mathbb{T}^{*} \times \mathbb{R}^{n}\right)$, holomorphic with respect to $z$ in $\Omega$. Put $A=\operatorname{Op}_{h}^{w}(-\nabla \lambda(k) \cdot r)$. Since $K_{2} \in S^{\delta+2}\left(\mathbb{T}^{*} \times \mathbb{R}^{n}\right) \subset S^{0}\left(\mathbb{T}^{*} \times \mathbb{R}^{n}\right)$, it follows from the $h$-pseudodifferential calculus and the Calderon-Vaillancourt's theorem that

$$
\left[\widetilde{\mathrm{P}}_{s}, A\right]=\mathrm{Op}_{\mathrm{h}}^{w}\left(\left|\nabla_{\mathrm{k}} \lambda(\mathrm{k})\right|^{2}-\left(-\mathrm{r} \nabla_{\mathrm{r}} \varphi(-\mathrm{r})\right) \cdot \Delta \lambda(\mathrm{k})\right)+\mathcal{O}(\mathrm{h}),
$$

in $\mathcal{L}\left(\mathrm{L}^{2}\left(\mathbb{T}^{*}\right)\right)$, uniformly for $s \in \Omega \cap \mathbb{R}$. Here $[\cdot, \cdot]$ denotes the commutator.

The assumption (A3) and the Gärding inequality (see [11, chapter 8]) imply that for $f \in$ $\mathcal{C}_{0}^{\infty}(\Omega \cap \mathbb{R})$ there exists $\mathrm{C}>0$ such that

$$
f\left(\widetilde{P}_{s}\right)\left[\widetilde{P}_{s}, A\right] f\left(\widetilde{P}_{s}\right) \geq \operatorname{Cf}\left(\widetilde{P}_{s}\right)^{2} \quad \text { for } h \text { small enough and uniformly for } s \in \Omega \cap \mathbb{R} .
$$

Now applying $[14$, Theorem 1$]$, we get, for all $l \in \mathbb{N}^{*}$,

$$
\left\|<h D_{k}>^{-\alpha}\left(\mu \pm i 0-\widetilde{P}_{s}\right)^{-l}<h D_{k}>^{-\alpha}\right\|=\mathcal{O}\left(h^{-l}\right), \text { for all } \alpha>l-\frac{1}{2},
$$

uniformly for $\mu \in[a, b]$ and $s \in \Omega \cap \mathbb{R}$. Take $s=\mu$ in (3.29) we obtain the estimation (3.28) for $j=1$.

Theorem 3.11 (Limiting absorption Theorem). With the same assumptions as Lemma 3.10. One has, for $\mathrm{l} \in \mathbb{N}^{*}$, there exists $\mathrm{h}_{\mathrm{O}}(\mathrm{l})>0$ small enough such that for all $\mathrm{O}<\mathrm{h}<\mathrm{h}_{\mathrm{O}}(\mathrm{l})$ :

$$
\left\|<h x>^{-\alpha}(P(h)-\mu \pm i 0)^{-l}<h x>^{-\alpha}\right\|_{\mathcal{L}\left(L^{2}\left(\mathbb{R}_{x}^{n}\right)\right)}=\mathcal{O}\left(h^{-l}\right),
$$

for all $\alpha>l-\frac{1}{2}$ and uniformly for $\mu \in[a, b]$.

Proof. Recall that the operator $\mathrm{P}(\mathrm{h})$ acting on $\mathrm{L}^{2}\left(\mathbb{R}^{n}\right)$ with domain $\mathrm{H}^{2}\left(\mathbb{R}^{n}\right)$ is unitary equivalent to

$$
\mathbb{P}_{1}(h):=\left(D_{y}+h D_{x}\right)^{2}+V(y)+\varphi(x),
$$

acting on $\mathbb{L}^{0}$ with domain $\mathbb{L}^{2}$, where $\mathbb{L}^{\mathrm{m}}:=\left\{u(x) \mathrm{T}_{\Gamma}(x, y) ; \partial_{\chi}^{\alpha} u \in \mathrm{L}^{2}\left(\mathbb{R}^{\mathrm{n}}\right), \forall \alpha,|\alpha| \leq \mathrm{m}\right\}$ for $\mathrm{m} \in \mathbb{N}$ and $\mathrm{T}_{\Gamma}$ is a distribution in $\mathcal{S}^{\prime}\left(\mathbb{R}^{2 \mathfrak{n}}\right)$ defined by

$$
T_{\Gamma}(x, y)=\frac{1}{\operatorname{vol}(E) h^{n}} \sum_{\beta^{*} \in \Gamma^{*}} e^{i(x-h y) \frac{\beta^{*}}{h}} .
$$

Here $E$ is a fundamental domain of $\Gamma$. Then we will prove (3.30) for $\mathbb{P}_{1}(\mathrm{~h})$.

It follows from identity (3.6) that:

$$
\begin{aligned}
\langle h y\rangle^{-\alpha}\left(\mathbb{P}_{1}(h)-z\right)^{-1}\langle h y\rangle^{-\alpha}= & \langle h y\rangle^{-\alpha} E_{1}(z, h)\langle h y\rangle^{-\alpha}-\langle h y\rangle^{-\alpha} E_{1,+}(z, h)\left\langle h D_{k}\right\rangle^{\alpha} . \\
& \cdot\left\langle h D_{k}\right\rangle^{-\alpha} E_{1,-+}(z, h)^{-1}\left\langle h D_{k}\right\rangle^{-\alpha} \cdot\left\langle h D_{k}\right\rangle^{\alpha} E_{1,-}(z, h)\langle h y\rangle^{-\alpha} .
\end{aligned}
$$


Since $E_{1}(z, h)$ is holomorphic then the first term of the r.h.s is bounded. On the other hand, as in Proposition 3.1, we prove that

$$
\begin{aligned}
&\left(\begin{array}{cc}
\langle h y\rangle^{-\alpha}\left(\mathbb{P}_{1}(h)-z\right)\langle h y\rangle^{\alpha} & \langle h y\rangle^{-\alpha} R_{+}^{*}\left\langle h D_{k}\right\rangle^{\alpha} \\
\left\langle h D_{k}\right\rangle^{-\alpha} R_{+}\langle h y\rangle^{\alpha} & 0
\end{array}\right)= \\
&\left(\begin{array}{cc}
\langle h y\rangle^{-\alpha} & 0 \\
0 & \left\langle h D_{k}\right\rangle^{-\alpha}
\end{array}\right)\left(\begin{array}{cc}
\mathbb{P}_{1}(h)-z & R_{+}^{*} \\
R_{+} & 0
\end{array}\right)\left(\begin{array}{cc}
\langle h y\rangle^{\alpha} & 0 \\
0 & \left\langle h D_{k}\right\rangle^{\alpha}
\end{array}\right)
\end{aligned}
$$

is well-defined as a bounded operator from $\mathbb{L}^{2} \times \mathrm{L}^{2}\left(\mathbb{T}^{*} ; \mathbb{C}^{\mathrm{N}}\right)$ to $\mathbb{L}^{0} \times \mathrm{L}^{2}\left(\mathbb{T}^{*} ; \mathbb{C}^{\mathrm{N}}\right)$ and is bijective with bounded two-sided inverse given by:

$$
\begin{aligned}
&\left(\begin{array}{cc}
\langle h y\rangle^{-\alpha} E_{1}(z, h)\langle h y\rangle^{\alpha} & \langle h y\rangle^{-\alpha} E_{1,+}(z, h)\left\langle h D_{k}\right\rangle^{\alpha} \\
\left\langle h D_{k}\right\rangle^{-\alpha} E_{1,-}(z, h)\langle h y\rangle^{\alpha} & \left\langle h D_{k}\right\rangle^{-\alpha} E_{1,-+}(z, h)\left\langle h D_{k}\right\rangle^{\alpha}
\end{array}\right)= \\
&\left(\begin{array}{cc}
\langle h y\rangle^{-\alpha} & 0 \\
0 & \left\langle h D_{k}\right\rangle^{-\alpha}
\end{array}\right)\left(\begin{array}{cc}
E_{1}(z, h) & E_{1,+}(z, h) \\
E_{1,-}(z, h) & E_{1,-+}(z, h)
\end{array}\right)\left(\begin{array}{cc}
\langle h y\rangle^{\alpha} & 0 \\
0 & \left\langle h D_{k}\right\rangle^{\alpha}
\end{array}\right) .
\end{aligned}
$$

Then $\langle h y\rangle^{-\alpha} E_{1,+}(z, h)\left\langle h D_{k}\right\rangle^{\alpha}$ and $\left\langle h D_{k}\right\rangle^{-\alpha} E_{1,-}(z, h)\langle h y\rangle^{\alpha}$ are well-defined and bounded. Therefore combining this with Lemma 3.10 we obtain Theorem 3.11 for $l=1$. With the same arguments we obtain the result for $l \geq 2$.

Remark 3.12. A simple consequence of Theorem 3.11 is that $\mathrm{P}(\mathrm{h})$ has no embedded eigenvalues in $[a, b]$.

\subsection{Proof of the pointwise asymptotic expansion of $\xi_{h}^{\prime}(\cdot)$}

Recall that $\Omega$ is a small complex neighborhood of $[a, b]$ such that the proposition 3.1 holds. For $s \in \Omega \cap \mathbb{R}$, we consider the $h$-pseudodifferential operator $B_{s}\left(k,-h D_{k} ; h\right):=H^{w}\left(k,-h D_{k} ; h\right)+$ $h^{2} G^{w}\left(k,-h D_{k} ; s, h\right)$ with the following symbol:

$$
b_{s}(k, r ; h)=H(k, r ; h)+h^{2} G(k, r ; s, h),
$$

where $\mathrm{H}(\mathrm{k}, \mathrm{r} ; \mathrm{h})$ given by $(3.26)$ and $\mathrm{G}(\cdot ; z, h) \in \mathrm{S}^{\delta+2}\left(\mathbb{T}^{*} \times \mathbb{R}^{n}\right)$, holomorphic with respect to $z$ in $\Omega$ (see Corollary 3.9).

Clearly, the principal symbol of $B_{s}$ (i.e. $b_{s}^{0}=\lambda(k)+\varphi(r)$ ) is independent on s. Moreover, the assumption (A3) implies that $[a, b]$ is a non-trapping region for the classical hamiltonian $b_{s}$. Now, by constructing a long-time parametrix for the time-dependent equation

$$
\left(h D_{t}-B_{s}\right) U(t)=0, \quad U(0)=I,
$$


we can apply the Robert-Tamura method [33, 34, 35] (see also [31, Remark 6.1]) to prove that

$$
\left[\operatorname{tr}\left(\left(z-\mathrm{B}_{\mathrm{s}}\right)^{-1}-(z-\lambda(\mathrm{k}))^{-1}\right)\right]_{z=\mu-\mathrm{i} 0}^{z=\mu+\mathrm{i} 0}
$$

has a complete asymptotic expansion in powers of $h$ uniformly for $\mu \in[a, b]$ and $s \in \Omega \cap \mathbb{R}$. Remembering (3.27) and take $s=\mu \in[a, b] \subset \Omega$ we obtain (2.8).

\section{Received: February 2011. Revised: March 2011.}

\section{References}

[1] S. Alama, P-A. Deift, and R. Hempel, Eigenvalue branches of the Schrödinger operator, Comm. Math. Phys. 121 (1989), no. 2, 291-321.

[2] M-Sh. Birman and D-R. Yafaev, The spectral shift function. the papers of M-G. Kreŭn and their further development, St. Petersburg Math. J. 4 (1993), no. 5, 833-870.

[3] , The scattering matrix for a perturbation of a periodic Schrödinger operator by decreasing potential, St. Petersburg Math. J. 6 (1995), no. 3, 453-474.

[4] V-S. Buslaev, Scattered plane waves, spectral asymptotics and trace formulae in exterior problems, (Russian), Dokl. Akad. Nauk SSSR 197 (1971), 999-1002.

[5] V-S. Buslaev, Semi-classical approximation for equations with periodic coefficients, Russian. Math. Surveys 42 (1987), no. 6, 97-125.

[6] Y. Colin de Verdière, Une formule de trace pour l'opérateur de Schrödinger dans $\mathbb{R}^{3}$, Ann. Ec. Normale Sup. 14 (1981), 27-39.

[7] M. Dimassi, Développements asymptotiques des perturbations lentes de l'opérateur de Schrödinger périodique, Comm. Partial Differential Equations 18 (1993), no. 5-6, 771-803. (1998), no. 1-2, 1-32.

[9] , Resonances for a slowly varying perturbation of a periodic Schrödinger operator, Canad. J. Math. 54 (2002), no. 5, 998-1037.

[10] Spectral shift function and resonances for slowly varying perturbations of periodic Schrödinger operators, J. Funct. Anal. 225 (2005), no. 1, 193-228.

[11] M. Dimassi and J. Sjöstrand, Spectral asymptotics in the semi-classical limit, London Mathematical Society Lecture Note Series, vol. 268, Cambridge University Press, Cambridge, 1999.

[12] M. Dimassi and M. Zerzeri, A local trace formula for resonances of perturbed periodic Schrödinger operators, J. Funct. Anal. 198 (2003), no. 3, 142-159. 
[13] C. Gérard, Resonances theory for periodic Schrödinger operators, Bull.Soc.Math. France 118 (1990), no. 1, 27-54.

[14] A proof of the abstract limiting absorption principle by energy estimates, J. Funct. Anal. 254 (2008), no. 11, 2707-2724.

[15] C. Gérard, A. Martinez, and J. Sjöstrand, A mathematical approach to the effective hamiltonian in perturbed periodic problems, Comm. Math. Phys. 142 (1991), 217-244.

[16] C. Gérard and F. Nier, Scattering theory for the perturbations of periodic Schrödinger operators, J. Math. Kyoto Univ. 38 (1998), 595-634.

[17] L. Guillopé, Asymptotique de la phase de diffusion pour l'opérateur de Schrödinger dans $\mathbb{R}^{n}$, Sém. E.D.P Ecole polytechnique, Exp. 5, 1984-1985.

[18] J-C. Guillot, J. Ralston, and E. Trubowitz, Semi-classical methods in solid state physics, Comm. Math. Phys. 116 (1988), 401-415.

[19] B. Helffer and J. Sjöstrand, Équation de Schrödinger avec champ magnétique et équation de harper, Schrödinger operators (Sønderborg, 1988), Lecture Notes in Physics, vol. 345, Springer, Berlin, 1989, pp. 118-197.

[20] On diamagnetism and de haas-van alphen effect, Ann. Inst. H. Poincaré Phys. Théor. 52 (1990), no. 4, 303-375.

[21] F. Hôvermann, H. Spohn, and S. Teufel, Semi-classical limit for the Schrödinger equation with a short scale periodic potential, Comm. Math. Phys. 215 (3) (2001), 609-629.

[22] M-G. Krĕn, On the trace formula in perturbation theory. (Russian), Mat. Sbornik N.S. 33 (1953), no. 75, 597-626.

[23] I-M. Lifshits, On a problem in perturbation theory. (Russian), Uspekhi Mat. Nauk 7 (1952), no. $1,171-180$.

[24] A. Majda, J. Ralston, An analogue of Weyl's Theorem for unbounded domains, Duke Math. J. 45 (1978), 183-196.

[25] V. Petkov, G. Popov, Asymptotic behavior of the scattering phase for non-trapping obstacles, Ann. Inst. Fourier Grenoble 32 (1982), 111-149.

[26] G. Popov, Asymptotic behavior of the scattering phase for Schrödinger operator, Publ. Acad. Scien. Sofia 35 (1982), 885-888.

[27] M. Reed and B. Simon, Methods of modern mathematical physics. IV, Academic Press, New York, 1978, Analysis of operators.

[28] D. Robert, Autour de l'approximation semi-classique, Progress in Mathematics, no. 68, Birkhäuser Boston, Inc., Boston, MA., 1987. 
[29]

, Asymptotique à grande énergie de la phase de diffusion pour un potentiel, Asymptotic Anal. 3 (1991), no. 4, 301-320.

[30]

, Asymptotique de la phase de diffusion à haute énergie pour des perturbations du second ordre du laplacien, Ann. Sci. école Norm. Sup. (4) 25 (1992), no. 2, 107-134.

, Relative time-delay for perturbations of elliptic operators and semiclassical asymptotics, J. Funct. Anal. 126 (1994), no. 1, 36-82.

[32] , Semiclassical asymptotics for the spectral shift function, Amer. Math. Soc. Transl. 189 (1999), no. 2, 187-203.

[33] D. Robert and H. Tamura, Semi-classical Bounds for Resolvents of Schrödinger Operators and Asymptotics for Scattering Phases, Comm. Partial Differential Equations 9 (1984), no. 10, 1017-1058. , Semiclassical estimates for resolvents and asymptotics for total scattering cross-sections, Ann. Henri Poincaré Phys. Théor. 46 (1987), no. 4, 415-442. , Semi-classical asymptotics for local spectral densities and time delay problems in scattering processes, J. Funct. Anal. 80 (1988), no. 1, 124-147.

[36] J-C. Slater, Electrons in perturbed periodic lattices, Phys. Rev. 76 (1949), 1592-1600.

[37] M-M. Skriganov, The spectrum band structure of the three-dimensional Schrödinger operator with periodic potential, Invent. Math. 80 (1985), no. 1, 107-121.

[38] , Geometric and arithmetic methods in the spectral theory of multidimensional periodic operators, Russian A translation of Trudy Mat. Inst. Steklov. 171 (1985), Proc. Steklov Inst. Math. 171 (1987), no. 2, vi+121 pp.

[39] J. Sjöstrand, Microlocal analysis for the periodic magnetic Schrödinger equation and related questions, Microlocal analysis and applications (Montecatini Terme, 1989), Lecture Notes in Math., vol. 1495, Springer, Berlin, 1991, pp. 237-332.

[40] M-A. Shubin, The spectral theory and the index of elliptic operators with almost periodic coefficients, Russian Math. Surveys 34 (1979), no. 2, 109-158.

[41] L-E. Thomas, Time dependent approach to scattering from impurities in a crystal, Comm. Math. Phys. 33 (1973), 335-343.

[42] D. Yafaev, Scattering theory: some old and new problems, Scattering theory: some old and new problems, Lecture Notes in Math., vol. 1737, Springer, Berlin, 2000, pp. xvi+169 pp. 